\title{
Peer Support for Women with Antenatal Depression
}

\section{Fiona Cust* and Ruth Carter}

School of Health and Social Care, Staffordshire University, Blackheath Lane, Stafford, UK

\section{Abstract}

Perinatal mental health is a major public health concern. Antenatal depression (AND) affects approximately $13 \%$ of women and is associated with psychological and physical morbidity, including poor birth outcomes and an increased risk of maternal suicide.

Qualitative studies suggest that women highly value peer support. Such studies have explored some of the mechanisms of peer support that have benefitted woman. These include being able to speak openly to a peer who has experienced similar feelings of alienation, of feeling abnormal, isolated, and experiencing stigma. Findings from these studies have included feelings of increased social support, confidence, selfesteem and hope for recovery.

The aim of this randomised controlled trial (RCT) was to assess whether the provision of support in the form of six weekly, one-hour visits from a PSW to women with antenatal depression (AND) was an effective and acceptable intervention with the potential to reduce AND.

\section{Introduction and Background}

Perinatal mental health is a major public health concern. Antenatal depression (AND) affects approximately $13 \%$ of women and is associated with psychological and physical morbidity, including poor birth outcomes and an increased risk of maternal suicide [1].

Mental health problems in pregnancy often need more urgent intervention than at other times. The potential effect on the fetus, and on the woman's physical health and care, and her ability to function and care for her family can be severely comprised [1]. Pharmacologic interventions during pregnancy are highly complex given the risks of fetal exposure to medication [2]. Women often do not like taking medication and this can mean they are afraid of admitting to any feelings of depression or low mood [3].This can understandably; affect their engagement in any potential treatment [4].

Mental health peer support is based upon the principle that people who have shared the experience of mental illness are uniquely able to relate to one another with empathy and validation [5]. The role of peer support worker is based upon the premise that people with similar experiences can authentically support others with similar issues [6,7]. Peer support may be mutual (between people who are currently unwell, in a group or an online forum) or unidirectional (between a trained peer supporter who has recovered and a recipient who is unwell). As a form of social support, it is often conceptualised as comprising emotional, appraisal (affirmation), and informational support [5]. Different forms of organised peer support have been used to help women experiencing, or at risk of experiencing, postnatal depression.

Qualitative studies suggest that women highly value peer support. Such studies have explored some of the mechanisms of peer support that have benefitted woman. These include being able to speak openly to a peer who has experienced similar feelings of alienation, of being abnormal, feeling isolated and experiencing stigma. Findings from these studies have included feelings of increased social support, confidence, self-esteem and hope for recovery $[5,8,9]$.

Pfeiffer et al. [10] state that additional studies are needed to determine the effectiveness of PSWs in primary care and other settings with limited mental health resources. Research has revealed that peer support for women with postnatal depression (PND) is effective, acceptable and valued by women with PND and also the peer support workers $[11,12]$.

However, it has been suggested that screening and support for perinatal mental health should begin in the antenatal period. NICE perinatal mental health guidelines [2] recommended that all pregnant women should be asked about their mental health using the Whooley questions, at their initial appointment with their midwife - thus enabling the recognition of potential problems early and signposting to relevant agencies.

The aim of this randomised controlled trial (RCT) was to assess whether the provision of support in the form of six weekly, one-hour visits from a PSW to women with antenatal depression (AND) was an effective and acceptable intervention with the potential to reduce AND.

\section{Methodology}

A RCT pilot study with 10 women allocated to the intervention group and 10 to the control group was conducted. Women were randomised anonymously by a computer generated package to ensure that members of the research team had no influence upon this process. Once a woman had been randomised they were fully consented by a member of the research team into the study and assigned a number. All data collected was allocated to the correct number for the woman in order to maintain anonymity. The intervention continued for a sixweek period with a one-hour visit scheduled by a PSW each week in a venue agreed by both the worker and the participant. The women allocated to the control group received the standard support offered by their Midwife and GP.

"Corresponding Author: Dr. Fiona Cust, Senior Lecturer-Children's Nursing Lead Placement Facilitator-Child, School of Health and Social Care, Staffordshire University, Blackheath Lane, Stafford, UK; E-mail: Fiona.Cust@staffs.ac.uk

Citation: Cust F, Carter R (2018) Peer Support for Women with Antenata Depression. Int J Nurs Clin Pract 5: 288. doi: https://doi.org/10.15344/23944978/2018/288

Copyright: (c) 2018 Cust et al. This is an open-access article distributed under the terms of the Creative Commons Attribution License, which permits unrestricted use, distribution, and reproduction in any medium, provided the original author and source are credited. 


\section{Results}

The results have been collated and are currently being peer reviewed for publication [5]. Following analysis of the data the women from the intervention group were contacted and invited to interview. Five women consented to a telephone interview, which was recorded and analysed using a thematic approach.

A number of themes emerged and, with consent, direct quotes will be utilised as examples.

\section{Talking therapy}

A number of the pregnant women described it as 'talking therapy'

Emily: 'I would just talk and talk and talk, sometimes I couldn't stop, it was just a relief to actually be able to talk to someone who just knew, you know, she just knew...'

Catherine: 'I didn't realise how much I needed to offload, it was like a big dam opening and everything just literally poured out, it was such a positive thing...'

\section{Safety}

A feeling of being 'safe' emerged during the interviews. The women described a feeling of calm and of feeling as if they were actually safe.

Caroline: 'Every time I venture out on my own I feel as if I'm being judged, as if people are looking at me and saying stuff. I don't go out anymore unless I'm with my Mum or my husband. But when I talk to my worker I suddenly feel an overwhelming feeling of being safe, as if I could go anyway and actually it would be ok.'

Jess: 'When I am with my worker I feel as if I will actually be ok, here is someone who actually gets it and understands, she makes me feel secure and as if I will be ok in the end.'

\section{Rationalise}

A number of the women shared the feeling of being rational, being able to see things in perspective

Rebecca: Because my worker had share similar feelings and worries, we were able to put it into perspective together. She just said, 'I felt like that too' and it was like a weight had been lifted. I just thought, phew, I'm not completely on my own then....'

Lucy: 'My worker gave me real answers, not just stuff from a text book, but lived experiences, her fears, her hopes, and I thought, yes, that's just it, I get it now.'

\section{Step back and reflect}

Some of the women discussed their fear of actually thinking about how they were feeling, of not wanting to take the time to consider how anxious they felt, how they dreaded having their baby and what a failure they felt they were being.

Caroline: My worker made me feel as if we are ok to stop and just think about how I felt and why - how could I help myself, what could I do. It was great to have that time to actually allow myself to say, ok you feel rubbish now why is that and what can you do about it.'
Emily: She allowed me to focus upon myself, as an individual, not just a pregnant lady. I could then look at myself and think about what was happening and why. It was almost as if I needed permission to do that and she gave that permission to me.'

\section{Shared stories and experiences}

Rebecca: 'It was a light bulb moment. My midwife is great but she just sort of ticks boxes and nods appropriately. She is nice but I always feel that she is achieving goals and exercises. My worker is so different. She tells me about how she felt and what she did that actually helped how she was feeling. Sometimes I listen and want to cry with relief as that is exactly how I have been feeling. It is just all about really getting it, simple really.'

Lucy-'It's not rocket science, it is a case of been there, seen that and done that. She tells me, 'oh yeah, I felt like that so I found this helped, see what you think'.... she just gets it really.'

\section{Discussion}

The aim of the study was to establish whether support provided by women with a previous experience of antenatal depression would assist and be acceptable to a woman with existing antenatal depression. This certainly does appear to have helped the participants receiving the support.

The women reported feelings of relief, being allowed to reflect and of the huge benefit of shared experiences. A single resounding factor appears to be that the peer support workers had experienced the same feelings and emotions and, subsequently, could offer guidance as to how to potentially help. The participants appeared to take great comfort from the fact that their worker had 'lived through the emotions and had come out of the other side. This gave them proof that they may do so too, a fact that many of them could not actually foresee when they were feeling so utterly despairing and helpless.

This study has added to the growing body of evidence that 'lived experience' is indeed invaluable - and that is exactly what the PSW provides

\section{Conclusion}

A further, larger, national study is currently being planned subject to funding being granted. It is envisaged that the pilot study will provide the evidence required that peer support does indeed have a positive impact upon a women's mental health and wellbeing within the antenatal period.

\section{Competing Interests}

The authors declare that they have no competing interests.

\section{References}

1. The Royal College of Midwives, 2015.

2. The National Institute for Health and Care Excellence, 2015.

3. Boath EH, Bradley E, Henshaw C (2004) Women's views of antidepressant in the treatment of postnatal depression. J Psychosom Obstet Gynaecol: 25: 221-233.

4. Flynn FJ, Reagans RE, Amanuatullah ET, Ames DR (2006) Helping one's way to the top: self-monitors achieve starts by helping others and knowing who helps whom. J Pers Soc Psychol 91: 1123-1137. 
Citation: Cust F, Carter R (2018) Peer Support for Women with Antenatal Depression. Int J Nurs Clin Pract 5: 288. doi: https://doi.org/10.15344/2394$4978 / 2018 / 288$

5. McLeish J, Redshaw M (2017) Mother's accounts of the impact of emotional wellbeing of organised peer support in pregnancy and early parenthood: a qualitative study. BMC Pregnancy Childbirth 17: 28.

6. Dennis CL (2010) Postpartum depression peer support: maternal perceptions from a RCT. International Journal of Nursing Studies 47: 560-568.

7. Davidson L, Chinman M, Sells D, Rowe M (2006) Peer Support among adults with serious mental illness: A report from the field. Schizophr Bull 32: 443450.

8. Carter R, Cust F, Boath E (2017) Peer Support Workers experiences of supporting women with postnatal depression: a constant comparative study. J Reprod Infant Psychol 36: 168-176.

9. Jones C, Jomeen J, Hayter M (2014) The impact of peer support in the context of perinatal mental illness: A meta-ethnography. Midwifery 30: 491-498

10. Pfeiffer PN, Heisler M, Piette JD, Rogers MA, Valenstien M, et al. (2011) Efficacy of peer support interventions for depression: a meta-analysis. General Hospital Psychiatry 33: 29-36.

11. Cust F (2016) Peer Support for mothers with postnatal depression: A Pilot Study. Community Pract 89: 38-41.

12. Cust F (2016) Peer Support for mothers with postnatal depression' The Journal of New Writing in Health and Social care 2: 21-31.

13. Howard L, Molyneaux E, Dennis CL, Rachat T, Stein A, Milgrom J (2015) Nonpsychoticmental disorders in the perinatal period. Lancet 384: 1775-1788. 